\title{
Body mass index increases the lymph node metastasis risk of breast cancer: a dose- response meta-analysis with 52904 subjects from 20 cohort studies
}

\author{
Junyi Wang ${ }^{1}$, Yaning Cai ${ }^{1}$, Fangfang Yu ${ }^{1}$, Zhiguang Ping ${ }^{1 *}$ (D) and Li Liu ${ }^{2^{*}}$
}

\begin{abstract}
Background: Since body mass index (BMI) is a convincing risk factor for breast cancer, it is speculated to be associated with lymph node metastasis. However, epidemiological studies are inconclusive. Therefore, this study was conducted to investigate the effect of BMI on the lymph node metastasis risk of breast cancer.

Methods: Cohort studies that evaluating BMI and lymph node metastasis in breast cancer were selected through various databases including PubMed, PubMed Central (PMC), Web of science, the China National Knowledge Infrastructure (CNKI), Chinese Scientific Journals (VIP) and Wanfang Data Knowledge Service Platform (WanFang) until November 30, 2019. The two-stage, random effect meta-analysis was performed to assess the dose-response relationship between BMI and lymph node metastasis risk. Between-study heterogeneity was assessed using $P^{2}$. Subgroup analysis was done to find possible sources of heterogeneity.

Results: We included a total of 20 studies enrolling 52,904 participants. The summary relative risk (RR) $(1.10,95 \% \mathrm{Cl}$ : 1.06-1.15) suggested a significant effect of BMI on the lymph node metastasis risk of breast cancer. The doseresponse meta-analysis $(R R=1.01,95 \% \mathrm{Cl}: 1.00-1.01)$ indicated a positive linear association between $\mathrm{BMI}$ and lymph node metastasis risk. For every $1 \mathrm{~kg} / \mathrm{m}^{2}$ increment of BMl, the risk of lymph node metastasis increased by $0.89 \%$. In subgroup analyses, positive linear dose-response relationships between BMI and lymph node metastasis risk were observed among Asian, European, American, premenopausal, postmenopausal, study period less than 5 years, and more than 5 years groups. For every $1 \mathrm{~kg} / \mathrm{m}^{2}$ increment of BMl, the risk of lymph node metastasis increased by 0.99 , $0.85,0.61,1.44,1.45,2.22$, and $0.61 \%$, respectively.
\end{abstract}

Conclusion: BMI significantly increases the lymph node metastasis risk of breast cancer as linear dose-response reaction. Further studies are needed to identify this association.

Keywords: Body mass index, Metastasis, Breast cancer, Dose-response relationship, Meta-analysis

\footnotetext{
*Correspondence: ping_zhg@163.com; liulixh@zzu.edu.cn

${ }^{1}$ College of Public Health, Zhengzhou University, No.100 Science Avenue,

Zhengzhou City 450001, Henan Province, China

${ }^{2}$ School of Basic Medical Sciences, Zhengzhou University, Zhengzhou, Henan, China
}

(c) The Author(s). 2020 Open Access This article is licensed under a Creative Commons Attribution 4.0 International License, which permits use, sharing, adaptation, distribution and reproduction in any medium or format, as long as you give appropriate credit to the original author(s) and the source, provide a link to the Creative Commons licence, and indicate if changes were made. The images or other third party material in this article are included in the article's Creative Commons licence, unless indicated otherwise in a credit line to the material. If material is not included in the article's Creative Commons licence and your intended use is not permitted by statutory regulation or exceeds the permitted use, you will need to obtain permission directly from the copyright holder. To view a copy of this licence, visit http://creativecommons.org/licenses/by/4.0/ The Creative Commons Public Domain Dedication waiver (http://creativecommons.org/publicdomain/zero/1.0/) applies to the data made available in this article, unless otherwise stated in a credit line to the data. 


\section{Background}

Breast cancer is one of the most common malignant tumors among females worldwide. According to the International Agency for Research on Cancer's GLOBOCAN 2018 [1], breast cancer was the second most common cancer only after lung cancer and the most frequent cancer among women with an estimated 2.09 million new cases diagnosed worldwide, making up $11.6 \%$ of all new cancer cases. Relative to cases, breast cancer ranked as the fourth cause of death from cancer overall (627 thousands), accounting for $6.6 \%$ of all cancer deaths. In China, it was estimated that there were 67,328 new breast cancer cases (16.3\% of all cancer cases) and 16, 178 deaths (7.8\% of all deaths) occurred in 2015 [2]. In addition, over the past decades, the prevalence of breast cancer is rising and getting younger gradually [3-5], which has caused serious economic burden and become an important global public health issue.

Although the rise in obesity and overweight showed some signs of leveling off, data from several countries indicated that obesity has become a worldwide epidemic [6]. Based on linear time trend analysis, a 33\% increase in obesity (body mass index, BMI $\geq 30 \mathrm{~kg} / \mathrm{m}^{2}$ ) prevalence was estimated, and obesity rates will be exceed $50 \%$ by 2030 [7]. It was regarded as a modifiable lifestyle risk factor for several chronic diseases in a growing body of literature, such as coronary heart disease [8], hypertension [9], type 2 diabetes mellitus [10], hyperlipidemia [11], stroke [12] and some cancers [13, 14]. Among them, several studies have found that overweight or obese women have an increased risk of breast cancer as compared to normal weight women, especially in postmenopausal women. A case-control study [15] conducted in Iran reported that obese postmenopausal women had a threefold increased risk of breast cancer (odds ratio, $O R=3.21,95 \%$ CI: 1.15-8.47). In a pooled analysis [16] of eight representative large-scale cohort studies, the increased risk of breast cancer with higher BMIs was confirmed among Japanese postmenopausal women. Yanzi Chen's [17] dose-response meta-analysis was performed on BMI and breast cancer incidence, which showed that the breast cancer risk increased by $3.4 \%$ for every $1 \mathrm{~kg} / \mathrm{m}^{2}$ increment of BMI in postmenopausal women. Furthermore, women who are obese with breast cancer diagnosis were reported to have greater disease mortality, higher recurrence rate and adverse overall and diseasefree survival $[18,19]$. So obesity also plays an important role in the prognosis of breast cancer.

Despite accumulated evidence that obesity may increase breast cancer risk, question remain, whether obesity is associated with lymph node metastasis, the most common form of metastasis in breast cancer? However, there was limited study focused on the relationship between obesity and lymph node metastasis in breast cancer, and the conclusions were inconsistent. For example, in a retrospective review of 1352 breast cancer patients [20], obese patients were more likely to have lymph node metastases compared with non-obese patients $(P=0.026)$. In another study [21] supporting this viewpoint, obesity was associated with increased number of involved axillary nodes $(P=0.003)$. On the contrary, Yadong Cui's [22] case series study found that there was no statistically significant association between BMI and axillary node involvement (adjusted $O R=1.28,95 \%$ CI: 0.90-1.81). Therefore, the present dose-response meta-analysis was conducted to investigate the association between obesity, as measured by BMI, and lymph node metastasis in breast cancer, and sub-analyses by different areas, menopausal status, study period were done to explore potential factors that influence the associations deeply.

\section{Methods \\ Search strategy}

In this study, we searched PubMed, PubMed Central (PMC), Web of science and Chinese academic databases including the China National Knowledge Infrastructure (CNKI), VIP database of Chinese Scientific Journals (VIP) and Wanfang Data Knowledge Service Platform (WanFang) for publications on the association between BMI and lymph node metastasis in breast cancer in humans up to November 30, 2019. The following combination of keywords was used to identify studies from electronic databases: (obesity OR "body mass index" OR BMI) AND ("breast cancer") AND ("metastasis"). To avoid missing any relevant studies, all reference lists of eligible articles and related reviews were searched for additional publications. We did not include unpublished documents and grey literature, such as conference abstracts, theses (including dissertations) and patents.

\section{Study selection}

Studies were included according to the following criteria: (1) full-text articles were available as Chinese or English language; (2) study design was a cohort study; (3) the height and weight of patients were measured at the time of diagnosis; (4) studies had BMI categories of no fewer than three, and provided the number of cases for each BMI category; (5) studies reported the metastasis type of patients, such as lymph node metastasis, positive lymph nodes and so on. If more than one publication of a given study exists, only the publication with higher number participants was included.

\section{Data extraction}

All potential relevant publications were inserted in EndNote X8 software. Then, qualified studies were obtained for full-text screening. After the final evaluation, the authors extracted and recorded the required data: name of the first author; year of publication; country of origin; age (range) of study population; study period; intervals 
of each BMI category; cases number of each category and so on.

\section{Quality assessment}

Using the Newcastle-Ottawa's Scale (NOS), the quality of the included studies were assessed. This scale ranges from 0 to 9 stars and awards four stars for selection of study participants, two stars for comparability of studies, and three stars for the adequate ascertainment of outcomes, and each item is assigned with a star if a study meets the criteria. We considered a study to be of high quality if its NOS score was more than six stars.

Study selection, data extraction, and quality assessment were done by two independent reviewers, and any controversies across selecting eligible articles were resolved by mutual discussion.

\section{Statistical analysis}

The relative risk $(R R)$ and its $95 \% C I$ were considered as the effect size of all studies. For the highest versus lowest category meta-analysis, the risk estimates for the highest compared with the lowest categories of BMI was combined using the DerSimonian and Laird random-effects model [23]. For the dose-response meta-analysis, the dosage value corresponding to each BMI was the median or mean of the upper and lower boundaries. When the lowest or the highest category was open-ended, we assumed that the open-ended interval length was same as the adjacent interval $[24,25]$.

For non-linear dose-response relation, the covarianceadjusted multiple variables regression model was used to estimate and test the overall effect of curvilinear doseresponses. For linear dose-response relationship, a slope for each study was estimated as the first step, then derived an overall estimates by weighted average of the individual slopes [26].

Heterogeneity among studies was assessed by I-square $\left(I^{2}\right)$ statistic. An $I^{2}$ above 50\% indicated high heterogeneity, and a random effect model was implemented. Predefined subgroup analyses based on area, menopausal status, study period and study population were conducted to detect potential sources of heterogeneity. To explore the influence of each study on the pooled effect size, a sensitivity analysis was used by omitting one study at a time. Publication bias was identified with the Begg's rank correlation test and Egger's regression test $[27,28]$. All statistical analyses were performed using Stata software version 14.0 (Stata Corp, College Station, TX, USA). Statistical significance level was set at $\alpha=0.05$, except publication bias or heterogeneity test with $\alpha=0.10$.

\section{Results}

\section{Literature screening results}

From the preliminary literature search, a total of 1141 articles were identified, with 9 references traced back.
After excluding 123 de-duplicated publications, we read 1027 titles and abstracts. Upon the exclusion of 965 clearly irrelevant records, we obtained 62 full-text articles for further assessment. Finally, a total of 20 articles were initially included in this meta-analysis. Among them, there were one Chinese article and 19 English articles. A detailed description of how studies were selected is presented in Fig. 1.

\section{Characteristics and quality assessment}

There were total 20 [29-48] articles included, all of which were cohort studies with a sample size of 52,904 people. Among the 20 studies, three studies were conducted in Asia, eight in Europe, eight in America and one from the International Breast Cancer Study Group, which covering the population from the whole world. Besides, four studies provided information on premenopausal and postmenopausal women separately, one study provided data on premenopausal women, and two studies provided data on postmenopausal women only. In terms of study period, there were six studies less than or equal to 5 years, and 14 studies more than 5 years. As for study population, two studies focused on triplenegative breast cancer (TNBC) patients. NOS scale was used to evaluate the included articles with score ranged from 6 to 8 . The characteristics and quality score of the individual studies are shown in Table 1.

\section{Highest versus lowest BMI meta-analysis}

In this study, we selected the $R R$ s corresponding to the highest BMI categories as the highest dose, and the RRs corresponding to the lowest BMI categories as the lowest dose. Heterogeneity among these 20 included articles was statistically significant $(P=0.022$, $\left.I^{2}=43.0 \%\right)$, and the random effect model was used for meta-analysis. The results showed that there was a link between BMI and the lymph node metastasis risk of breast cancer, with a summary $R R$ of 1.10 (95\%CI: 1.06-1.15) (Fig. 2).

\section{Subgroup analyses}

When subgroup analyses were done for different areas, the results showed significant associations between BMI and lymph node metastasis of breast cancer in Asian $(R R=1.18, \quad 95 \% C I: \quad 1.08-1.30)$, European $(R R=1.08$, 95\%CI: $1.05-1.12)$ and American $(R R=1.13,95 \% C I$ : $1.04-1.23)$ women. Interestingly, there were positive associations both in the premenopausal women $(R R=1.12$, 95\%CI: 1.04-1.20) and postmenopausal women $(R R=$ 1.28, 95\%CI: 1.14-1.44). Besides, we conducted a subgroup analysis stratified by study period, the $R R(1.31$, $95 \% C I, 1.14-1.50)$ of less than and equal to 5 years was prominent higher than that of more than 5 years $(R R=$ 1.07, 95\%CI: 1.05-1.10). For study population, positive 


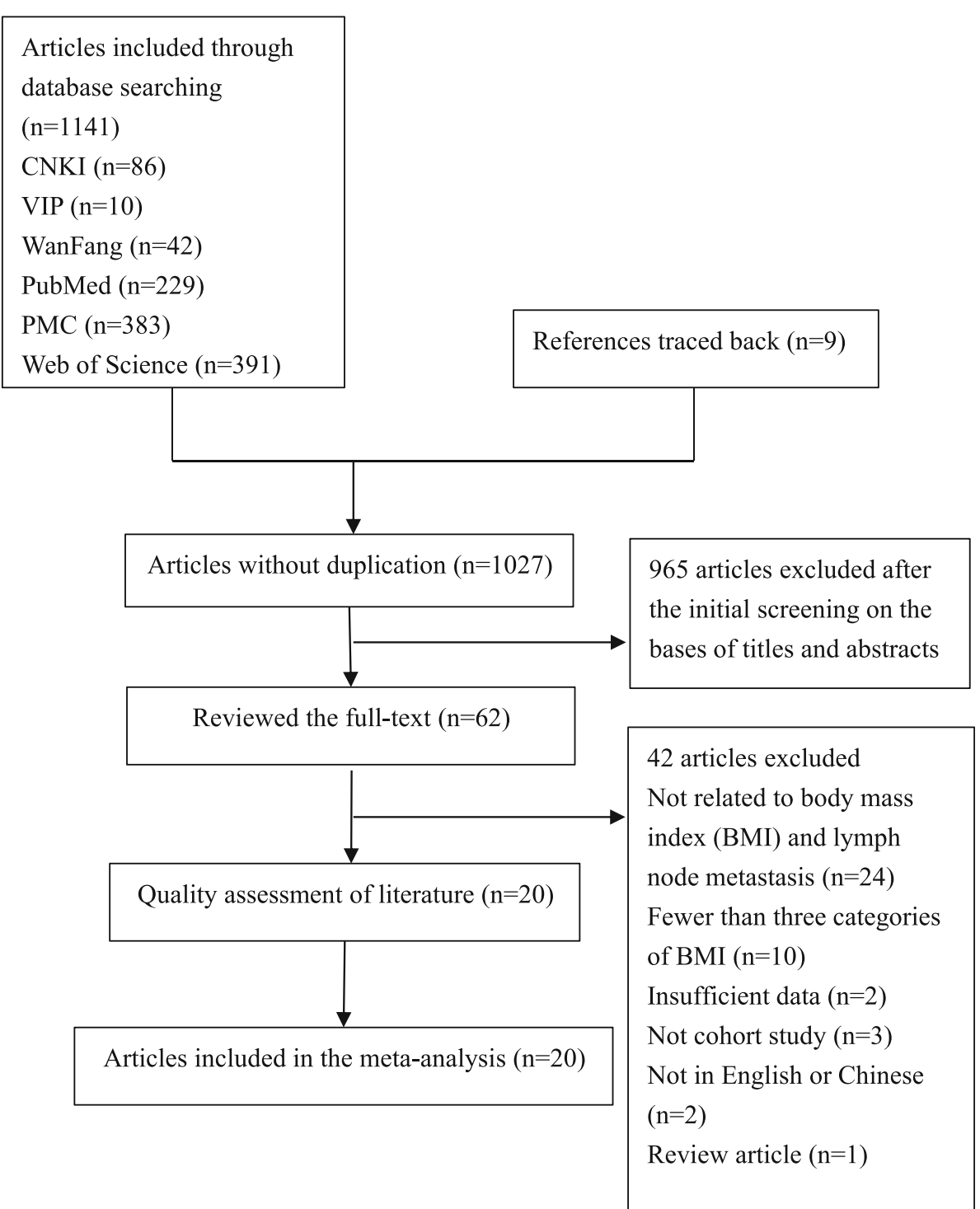

Fig. 1 Flow chart of literature retrieval and selection for this meta-analysis (CNKI: China National Knowledge infrastructure; VIP: VIP database of Chinese Scientific Journal; WanFang: Wanfang Data Knowledge Service Platform; PMC: PubMed Central)

significant associations between BMI and lymph node metastasis were observed in non-TNBC $(R R=1.08$, 95\%CI: 1.06-1.11), while poor association in TNBC patients $(R R=1.15,95 \% C I: 0.88-1.49)$. The subgroup analyses are shown in Table 2.

\section{Dose-response analyses}

Figure 3 showed the results of linear and nonlinear dose-response analysis of BMI and relative risk of lymph node metastasis in breast cancer. Firstly, we conducted a regression model test $(P=0.465)$, which showed no nonlinear dose-response relationship between BMI and lymph node metastasis. Secondly, linear dose-response regression model was used to test the relationship. The goodness of fit test $\left(x^{2}=30.34\right.$,
$P=0.048$ ) showed there was heterogeneity among the studies, and the random-effect model was used for the meta-analysis. Regression model test $\left(\chi^{2}=29.30\right.$, $P<0.001)$ revealed a positive linear dose-response association between BMI and lymph node metastasis. The results $(R R=1.01,95 \% C I: 1.00-1.01)$ showed that for every $1 \mathrm{~kg} / \mathrm{m}^{2}$ increment of $\mathrm{BMI}$, the risk of lymph node metastasis increased by $0.89 \%$.

The detailed information of the dose-response metaanalysis and subgroup analyses are shown in Table 3. In subgroup analyses, the results showed that the linear dose-response relationship between BMI and lymph node metastasis in Asian ( $R R=1.01,95 \% C I: 1.00-1.02)$, European $(R R=1.01,95 \% C I: 1.00-1.01)$, American $(R R=$ 1.01, 95\%CI: 1.00-1.01), premenopausal $(R R=1.01$, 
Table 1 The characteristics of studies included in this meta-analysis

\begin{tabular}{|c|c|c|c|c|c|c|c|c|}
\hline Author & Year & Country & Age (range) & $\begin{array}{l}\text { Study } \\
\text { period }\end{array}$ & The categories of BMI & $\begin{array}{l}\text { The number of } \\
\text { metastatic tumors }\end{array}$ & $\begin{array}{l}\text { The number of non- } \\
\text { metastatic tumors }\end{array}$ & NOS \\
\hline Xiaoyao Zhang & 2014 & China & $53(27-92)$ & $\begin{array}{l}2010.1- \\
2012.11\end{array}$ & $\begin{array}{c}\text { BMI <18.5 (underweight)/ } \\
18.5-22.9 \text { (normal)/ } \\
23-24.9 \text { (overweight)/ } \\
25-29.9 \text { (obese)/ } \\
\text { BMI } \geq 30 \text { (severe obese) }\end{array}$ & $2 / 27 / 21 / 85 / 25$ & $7 / 56 / 51 / 115 / 35$ & 6 \\
\hline Nicoletta Biglia & 2013 & Italy & $45 / 65$ & $\begin{array}{l}1999.1- \\
2009.12\end{array}$ & $\begin{array}{c}\mathrm{BMI}<19 \text { (underweight)/ } \\
19-24.9 \text { (normal)/ } \\
25-29.9 \text { (overweight)/ } \\
\text { BMI } \geq 30 \text { (obese) }\end{array}$ & $\begin{array}{c}\text { 20/141/49/29 } \\
\text { (premenopausal) } \\
\text { 20/247/217/97 } \\
\text { (postmenopausal) }\end{array}$ & $\begin{array}{c}\text { 37/200/44/20 } \\
\text { (premenopausal) } \\
35 / 372 / 243 / 125 \\
\text { (postmenopausal) }\end{array}$ & 7 \\
\hline $\begin{array}{l}\text { Orsolya } \\
\text { Hankó-Bauer }\end{array}$ & 2017 & Romania & $\begin{array}{c}58.29(27-80) \\
52.81 / 60.38 / \\
62.8\end{array}$ & $2012-2015$ & $\begin{array}{c}\mathrm{BMI}<25 \text { (normal weight)/ } \\
25-29.9 \text { (overweight)/ } \\
\text { BMI } \geq 30 \text { (obese) }\end{array}$ & $32 / 40 / 40$ & $54 / 48 / 31$ & 6 \\
\hline Ahmad Kaviani & 2013 & Iran & $49.62(21-88)$ & 2003-2011 & $\begin{array}{c}\text { BMI }<24.9 \text { (normal weight)/ } \\
25<\text { BMl }<29.9 \text { (overweight)/ } \\
\text { BMl }<\text { BMI30 (obese) }\end{array}$ & $\begin{array}{c}\text { 64/77/42 } \\
\text { (premenopausal) } \\
45 / 68 / 60 \\
\text { (postmenopausal) }\end{array}$ & $\begin{array}{c}\text { 60/52/22 } \\
\text { (premenopausal) } \\
\text { 39/70/31 } \\
\text { (postmenopausal) }\end{array}$ & 7 \\
\hline O.Keskin & 2013 & Turkey & $\begin{array}{l}48.9 \pm 10.7 \\
44.5 \pm 11.1 / \\
49.6 \pm 11.1 / \\
52.7 \pm 10.0\end{array}$ & $2001-2011$ & $\begin{array}{c}\text { 20-24.9 (normal weight)/ } \\
\text { 25-29.9 (overweight)/ } \\
\text { BMI } \geq 30 \text { (obese) }\end{array}$ & $231 / 266 / 226$ & $198 / 205 / 169$ & 7 \\
\hline $\begin{array}{l}\text { Geoffrey A. } \\
\text { Porter }\end{array}$ & 2006 & Canada & $60 \pm 15.5$ & $\begin{array}{l}2002.2 .15- \\
2004.2 .15\end{array}$ & $\begin{array}{c}\text { BMI <25 (normal/underweight)/ } \\
25-29.9 \text { (overweight)/ } \\
\text { BMI } \geq 30 \text { (obese/severely obese) }\end{array}$ & $36 / 33 / 46$ & $130 / 144 / 130$ & 8 \\
\hline $\begin{array}{l}\text { Marianne } \\
\text { Ewertz }\end{array}$ & 2011 & Denmark & -- & $1977-2006$ & $\mathrm{BMI}<25 / 25-29 / 30+$ & $6867 / 3201 / 1489$ & $4621 / 1937 / 849$ & 7 \\
\hline $\begin{array}{l}\text { Vincent C. } \\
\text { Herlevic }\end{array}$ & 2015 & US & $\begin{array}{l}61.360 .5 / \\
61.7 / 61.3\end{array}$ & 1997-2013 & $\begin{array}{c}\text { BMI<25 (normal weight)/ } \\
25-30 \text { (overweight)/ } \\
\text { BMl>30 (obese) }\end{array}$ & $40 / 71 / 142$ & $47 / 79 / 144$ & 8 \\
\hline $\begin{array}{l}\text { Marian L. } \\
\text { Neuhouser }\end{array}$ & 2016 & US & $50-79$ & 1993-1998 & $\begin{array}{c}\text { BMI<25 (normal weight)/ } \\
25-30 \text { (overweight)/ } \\
\text { 30-35 (obese, Grade 1)/ } \\
\text { BMI } \geq 35 \text { (obese, Grade 2+3) }\end{array}$ & $\begin{array}{c}\text { 168/245/184/138 } \\
\text { (postmenopausal) }\end{array}$ & $\begin{array}{l}\text { 579/825/547/345 } \\
\text { (postmenopausal) }\end{array}$ & 8 \\
\hline G. Berclaz & 2004 & $\begin{array}{l}\text { International } \\
\text { Breast } \\
\text { Cancer Study } \\
\text { Group }\end{array}$ & $\begin{array}{c}48(21-84) / \\
53(25-80) / \\
55(26-80)\end{array}$ & 1978-1993 & $\begin{array}{c}\text { BMI<24.9 (normal weight)/ } \\
25.0-29.9 \text { (intermediate)/ } \\
\text { BMI } \geq 30.0 \text { (obese) }\end{array}$ & $2613 / 1652 / 833$ & 695/386/191 & 6 \\
\hline $\begin{array}{l}\text { Vito Michele } \\
\text { Garrisi }\end{array}$ & 2012 & Italy & -- & $2004-2006$ & $\begin{array}{l}\text { BMl }<24.9 \text { (normal)/ } \\
25-29.99 \text { (overweight)/ } \\
\text { BMI } \geq 30 \text { (obese) }\end{array}$ & $43 / 63 / 38$ & $63 / 38 / 24$ & 6 \\
\hline $\begin{array}{l}\text { Luca } \\
\text { Mazzarella }\end{array}$ & 2013 & $\begin{array}{l}\text { European } \\
\text { Institute of } \\
\text { Oncology }\end{array}$ & --- & 1995-2005 & $\begin{array}{c}\text { BMI }<25 \text { (under/normal weight)/ } \\
25-29.99 \text { (overweight)/ } \\
\text { BMI } \geq 30 \text { (obese) }\end{array}$ & $\begin{array}{c}\text { 258/77/28 (ER } \\
\text { positive) } 149 / 66 / 29 \\
\text { (ER negative) }\end{array}$ & $\begin{array}{c}\text { 283/67/31 (ER } \\
\text { positive) } 159 / 63 / 18 \\
\text { (ER negative) }\end{array}$ & 7 \\
\hline Amelia Smith & 2018 & US & $67(63,73)$ & 1993-2009 & $\begin{array}{c}\mathrm{BMI}<18.5 \text { (underweight)/ } \\
18.5-24.9 \text { (normal weight)/ } \\
25-29.9 \text { (overweight)/ } \\
\text { BMI } \geq 30 \text { (obese) }\end{array}$ & $\begin{array}{c}\text { 3/282/261/197 } \\
\text { (postmenopausal) }\end{array}$ & $\begin{array}{l}\text { 19/869/819/561 } \\
\text { (postmenopausal) }\end{array}$ & 6 \\
\hline Kang Wang & 2019 & China & $\begin{array}{l}50.0 \pm 11.2 \\
48.5 \pm 13.7 / \\
49.1 \pm 11.1 / \\
52.6 \pm 10.7\end{array}$ & $\begin{array}{l}2005.1- \\
2015.12\end{array}$ & $\begin{array}{c}\text { BMl<18.5 (underweight)/ } \\
18.5-24.9 \text { (normal weight)/ } \\
\text { BMI } \geq 25 \text { (overweight and obese) }\end{array}$ & $\begin{array}{c}\text { 114/1644/537 } \\
\text { (premenopausal) } \\
70 / 1120 / 627 \\
\text { (postmenopausal) }\end{array}$ & $\begin{array}{c}\text { 100/1316/422 } \\
\text { (premenopausal) } \\
107 / 1184 / 559 \\
\text { (postmenopausal) }\end{array}$ & 6 \\
\hline E.R. Copson & 2014 & UK & $\begin{array}{c}36(18-40) 36 \\
(18-40) / 37 \\
(18-40) / 37 \\
(24-40)\end{array}$ & $2000-2008$ & $\begin{array}{c}\text { BMI }<25 \text { (under/healthy weight)/ } \\
25-30 \text { (overweight)/ } \\
\text { BMI } \geq 30 \text { (obese) }\end{array}$ & $\begin{array}{c}\text { 736/419/284 } \\
\text { (premenopausal) }\end{array}$ & $\begin{array}{c}\text { 766/354/236 } \\
\text { (premenopausal) }\end{array}$ & 7 \\
\hline $\begin{array}{l}\text { Aruna } \\
\text { Kamineni }\end{array}$ & 2013 & US & $64.5(40-93)$ & $\begin{array}{l}\text { 1988.1.1- } \\
\text { 1993.12.31 }\end{array}$ & $\begin{array}{l}\text { BMI }<25 \text { (normal weight)/ } \\
25-30 \text { (overweight)/ } \\
\text { BMI } \geq 30 \text { (obese) }\end{array}$ & $32 / 27 / 12$ & $174 / 102 / 66$ & 6 \\
\hline
\end{tabular}


Table 1 The characteristics of studies included in this meta-analysis (Continued)

\begin{tabular}{|c|c|c|c|c|c|c|c|c|}
\hline Author & Year & Country & Age (range) & $\begin{array}{l}\text { Study } \\
\text { period }\end{array}$ & The categories of BMI & $\begin{array}{l}\text { The number of } \\
\text { metastatic tumors }\end{array}$ & $\begin{array}{l}\text { The number of non- } \\
\text { metastatic tumors }\end{array}$ & NOS \\
\hline Ronny Mowad & 2013 & US & $\begin{array}{l}49.853 .2 / \\
49.1 / 49.3\end{array}$ & $\begin{array}{l}1998.3- \\
2011.9\end{array}$ & $\begin{array}{c}\text { BMI<25 (normal/underweight)/ } \\
25-29.9 \text { (overweight)/ } \\
\text { BMl }>30 \text { (obese) }\end{array}$ & 9/18/47 & $15 / 24 / 70$ & 8 \\
\hline $\begin{array}{l}\text { Foluso O. } \\
\text { Ademuyiwa }\end{array}$ & 2011 & US & $\begin{array}{l}54(26-92) \\
52.9 / 56.3 / \\
56.1\end{array}$ & $\begin{array}{l}1996.7- \\
2010.7\end{array}$ & $\begin{array}{c}\text { BMI } \leq 24.9 \text { (normal/underweight)/ } \\
25-29.9 \text { (overweight)/ } \\
\text { BMl>30 (obese) }\end{array}$ & $44 / 49 / 68$ & $80 / 81 / 96$ & 7 \\
\hline $\begin{array}{l}\text { Shaheenah } \\
\text { Dawood }\end{array}$ & 2008 & US & $\begin{array}{l}46(23-76) / \\
48(23-78) / \\
52(28-78)\end{array}$ & $1974-2000$ & $\begin{array}{c}\text { BMI } \leq 24.9 \text { (normal/underweight)/ } \\
25-29.9 \text { (overweight)/ } \\
\text { BMI } \geq 30 \text { (obese) }\end{array}$ & 186/175/186 & 21/19/16 & 7 \\
\hline Ozan Yazici & 2015 & Turkey & $48(18-92)$ & $\begin{array}{l}2002.1- \\
2013.10\end{array}$ & $\begin{array}{l}\text { 18.5-24.9 (normal weight)/ } \\
\text { 25-29.9 (overweight)/ } \\
\text { BMI } \geq 30.0 \text { (obese) }\end{array}$ & $\begin{array}{c}\text { 20/14/7 } \\
\text { (premenopausal) } 7 / \\
5 / 10 \\
\text { (postmenopausal) }\end{array}$ & $\begin{array}{c}\text { 549/393/226 } \\
\text { (premenopausal) } \\
\text { 228/419/409 } \\
\text { (postmenopausal) }\end{array}$ & 7 \\
\hline
\end{tabular}

BMI Body mass index, NOS Newcastle-Ottawa's Scale

95\%CI: 1.00-1.03), postmenopausal $(R R=1.01,95 \% C I$ : $1.01-1.02)$, study period $\leq 5$ years $(R R=1.02,95 \% C I$ : $1.01-1.03)$, study period $>5$ years $(R R=1.01,95 \% C I$ : $1.00-1.01)$ patients were statistically significant, and the risk increased by $0.99,0.85,0.61,1.44,1.45,2.22$, and $0.61 \%$, respectively. And the results of other two subgroups (TNBC and non-TNBC) were missing because of too small sample size in TNBC.

\section{Sensitivity analysis}

For the sensitivity analysis, we omitted one study at a time in turn to assess the potential studies which may influence the main results. The pooled $R R$ s indicated little variation ranging from 1.09 (95\%CI, 1.05-1.13) to 1.13 (95\%CI, 1.06-1.19), and the result was not influenced by any single study, indicating that the metaanalysis result was stable.

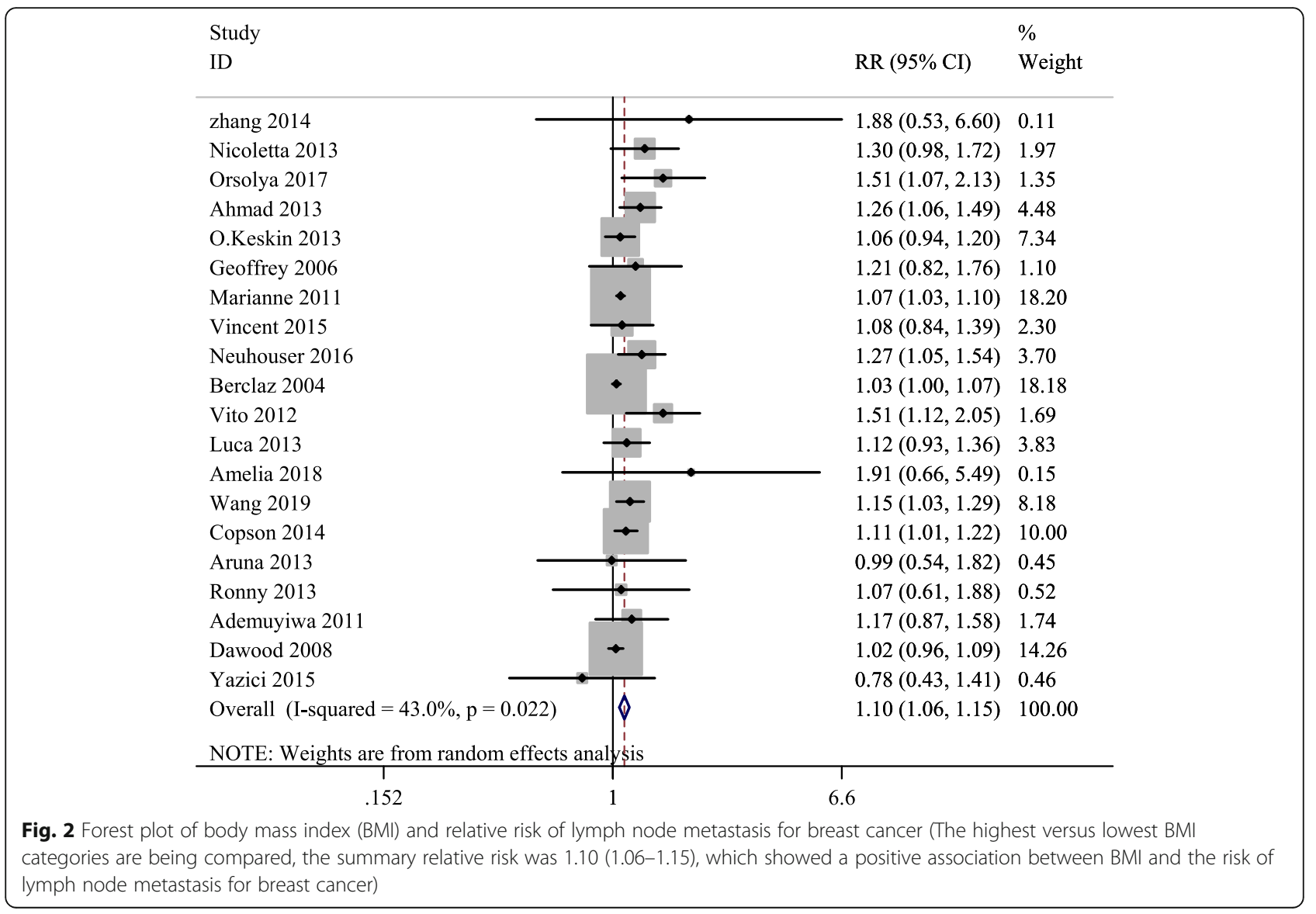


Table 2 Subgroup analyses showing difference between studies included in the meta-analysis (highest versus lowest BMI)

\begin{tabular}{|c|c|c|c|c|c|c|c|}
\hline \multirow[t]{2}{*}{ Variables } & \multirow{2}{*}{$\begin{array}{l}\text { Number } \\
\text { of } \\
\text { studies }\end{array}$} & \multirow{2}{*}{$\begin{array}{l}\text { Number } \\
\text { of cases }\end{array}$} & \multirow{2}{*}{$\begin{array}{l}\text { Pooled RR } \\
(95 \% \mathrm{Cl})\end{array}$} & \multicolumn{2}{|c|}{ Test of heterogeneity } & \multicolumn{2}{|l|}{ Publication bias } \\
\hline & & & & $\overline{P^{2}(\%)}$ & $P$ value & Begg's $P$ value & $\overline{\text { Egger's } P \text { value }}$ \\
\hline All & 20 & 30938 & $1.10(1.06,1.15)$ & 43.0 & 0.022 & 0.538 & 0.003 \\
\hline \multicolumn{8}{|l|}{ Area } \\
\hline Asia & 3 & 2968 & $1.18(1.08,1.30)$ & 0.0 & 0.555 & 1.000 & 0.339 \\
\hline Europe & 8 & 19791 & $1.08(1.05,1.12)$ & 44.6 & 0.082 & 0.266 & 0.116 \\
\hline America & 8 & 3847 & $1.13(1.04,1.23)$ & 47.4 & 0.065 & 0.902 & 0.079 \\
\hline \multicolumn{8}{|l|}{ Menopausal } \\
\hline Pre & 5 & 4291 & $1.12(1.04,1.20)$ & 31.6 & 0.211 & 0.806 & 0.489 \\
\hline Post & 6 & 4479 & $1.28(1.14,1.44)$ & 0.0 & 0.865 & 0.452 & 0.656 \\
\hline \multicolumn{8}{|l|}{ Study period } \\
\hline$\leq 5 y$ & 6 & 2250 & $1.31(1.14,1.50)$ & 0.0 & 0.709 & 0.707 & 0.860 \\
\hline$>5 y$ & 14 & 28688 & $1.07(1.05,1.10)$ & 26.8 & 0.167 & 0.743 & 0.051 \\
\hline \multicolumn{8}{|c|}{ Study population } \\
\hline TNBC & 2 & 429 & $1.15(0.88,1.49)$ & 0.0 & 0.789 & 1.000 & --- \\
\hline Non-TNBC & 18 & 30539 & $1.08(1.06,1.11)$ & 48.2 & 0.012 & 0.363 & 0.003 \\
\hline
\end{tabular}

TNBC Triple-negative breast cancer

\section{Publication bias}

No publication bias was found for subgroup analyses, except for the overall studies using Egger's test $(P=$ $0.003)$ and studies on non-TNBC patients using Egger's test $(P=0.003)$.

\section{Discussions}

Dose-response meta-analysis results showed that there was a linear dose-response relationship between BMI and lymph node metastasis in breast cancer. For every 1 $\mathrm{kg} / \mathrm{m}^{2}$ increment of BMI, the risk of lymph node

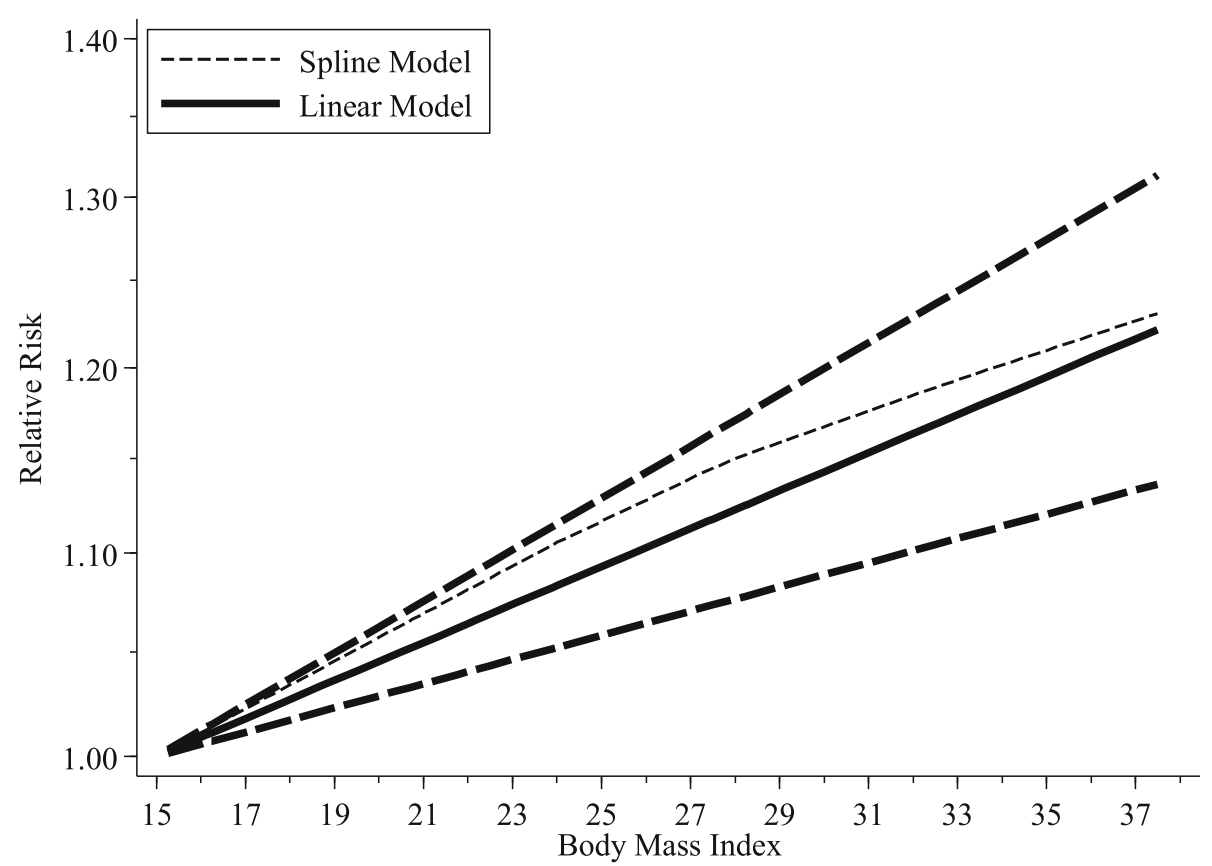

Fig. 3 The linear association between body mass index (BMI) and lymph node metastasis for breast cancer (The solid line and the dash line represent the estimated relative risk (RR) and its $95 \%$ confidence interval (CI) for the fitted linear trend. Lines with short dashes represent the nonlinear trend analysis result) 
Table 3 The results of linear dose-response analysis between body mass index (BMI) and lymph node metastasis of breast cancer

\begin{tabular}{|c|c|c|c|c|c|}
\hline Variables & Number of cases & Test of heterogeneity & Model & Regression model test & $R R(95 \% \mathrm{Cl})$ \\
\hline All & 52904 & $x 2=30.34, P=0.048$ & RE & $x 2=29.30, P<0.001$ & $1.0089(1.0057,1.0122)$ \\
\hline \multicolumn{6}{|l|}{ Area } \\
\hline Asia & 8854 & $X 2=4.71, P=0.095$ & FE & $x 2=11.13, P=0.001$ & $1.0099(1.0041,1.0157)$ \\
\hline Europe & 28979 & $x 2=13.70, P=0.057$ & FE & $x 2=36.31, P<0.001$ & $1.0085(1.0057,1.0113)$ \\
\hline America & 8701 & $X 2=5.16, P=0.641$ & FE & $x 2=6.01, P=0.014$ & $1.0061(1.0012,1.0110)$ \\
\hline \multicolumn{6}{|l|}{ Menopausal } \\
\hline Pre & 8994 & $x 2=10.53, P=0.032$ & RE & $X 2=5.61, P=0.018$ & $1.0144(1.0025,1.0264)$ \\
\hline Post & 12456 & $X 2=2.57, P=0.766$ & FE & $x 2=23.48, P<0.001$ & $1.0145(1.0086,1.0204)$ \\
\hline \multicolumn{6}{|c|}{ Study period } \\
\hline$\leq 5 y$ & 4901 & $X 2=3.66, P=0.600$ & FE & $\times 2=19.94, P<0.001$ & $1.0222(1.0124,1.0321)$ \\
\hline$>5 y$ & 48003 & $x 2=16.61, P=0.218$ & FE & $\times 2=40.88, P<0.001$ & $1.0061(1.0042,1.0080)$ \\
\hline
\end{tabular}

$R E$ Random effect, $F E$ Fixed effect

metastasis increased by $0.89 \%$. After grouping by areas, no significant geographical variation was detected, and the risk of lymph node metastasis increased by 0.99 , 0.85 , and $0.61 \%$ for every $1 \mathrm{~kg} / \mathrm{m}^{2}$ increment of BMI in Asian, European, and American women, respectively. Higher proportions of overweight and obese black or African-American breast cancer patients in the United States were mentioned in Ronny's study [45] and some other researches [49], which also tended to have poorer outcomes than white patients. An observation study of 223,895 women diagnosed with invasive breast cancer classified all patients into 8 race/ethnic groups including non-Hispanic white, Hispanic white, black, Chinese, Japanese, south Asian, other Asian, and other ethnicity [50]. Black women were significantly more likely to present with lymph node metastases than non-Hispanic white women $(24.1 \%$ vs $18.4, P<0.001)$, and lower probability was observed in Japanese women $(14.6 \%$ vs $18.4 \%, P<$ $0.001)$. Whether this race/ethnicity disparity existed when BMI were assessed remained unknown, although confounding factors, such as socioeconomic status and treatment imbalance, contributed in part. Also, in Chinese Han women, a possible interaction between Interleukin-18-137G/C, -607G/T polymorphisms and $\mathrm{BMI}$ in breast cancer patients was identified [51]. Overweight and obese $\left(B M I \geq 24 \mathrm{~kg} / \mathrm{m}^{2}\right)$ patients with $\mathrm{G} / \mathrm{T}$ genotype had a 5.45 -fold $(95 \% C I, 1.74-17.06)$ increased risk of lymph node metastasis relative to those with $\mathrm{T} / \mathrm{T}$ homozygotes. Subgroup analyses grouped by race/ethnicity or genotype would be more accurate to explore the linkage between obesity and lymph node metastasis in breast cancer, unfortunately, which was not available in the selected studies.

Besides, the lymph node metastasis risk of breast cancer with BMI in premenopausal women $\left(1.44 \% / 1 \mathrm{~kg} / \mathrm{m}^{2}\right)$ was similar to that in postmenopausal women $(1.45 \% / 1 \mathrm{~kg} /$ $\mathrm{m}^{2}$ ). In postmenopausal patients, obese women would have a high concentration of circulating estrogen, since most estrogen is produced in the adipose tissue [52]. Moreover, in the peripheral adipose tissue, obese women have a high activity of aromatase enzyme, which converts androstenedione to estrogen and testosterone to estradiol in turn stimulated by both interleukin-6 (IL-6) and tumor necrosis factor- $\alpha$ (TNF- $\alpha$ ) [53]. Elevated levels of estradiol are important to the development and growth of breast cancer, including lymph node metastasis, which are consistent with our results that shown increasing lymph node metastasis risk with BMI in postmenopausal women. Conversely, among premenopausal patients, systemic levels of estrogens are mainly produced by the ovaries, so not influenced by peripheral aromatization. It seems that obesity is not a independent factor in carcinogenesis and tumor metastasis in young breast cancer patients. Nevertheless, BMI was associated with a increased incidence for triplenegative subtype, but no association was shown in postmenopausal patients [54]. Similar findings also indicated that the association between obesity and TNBC was significant only among premenopausal women [55]. In addition to TNBC patients tended to present higher disease grade, more aggressive course, and high rate of recurrences [56], which may partly explained our results of similar lymph node metastasis risk in premenopausal and postmenopausal women. Due to small sample size in TNBC, subgroup analysis were not be conducted, as well as the interaction between triple-negative subtype and menopausal status. On the other hand, estrogen receptor (ER) positive in obese women also associated with menopausal status, although remained a matter of controversy in different studies [57, 58]. Only one included study [40] demonstrated results with ER positive and ER negative separately, and subgroup analysis was also failed.

When subgroup analysis was done for study period, it should be noted that a prominent increased risk $(2.22 \%$ / $1 \mathrm{~kg} / \mathrm{m}^{2}$ ) of lymph node metastasis with BMI occurred 
in less than 5 years compared with more than 5 years $\left(0.61 \% / 1 \mathrm{~kg} / \mathrm{m}^{2}\right)$. A possible explanation is the apparent older participants (Table 1) in three included studies $[34,37,44]$ followed less than 5 years, which constitutes approximately $80 \%$ of the subgroup patients. Another explanation is the substantial proportions (57-75\%) of overweight and obese patients distributed in this subgroup, especially in large sample size study (75\%) [37], which mainly resulted in higher lymph node metastasis risk in breast cancer patients.

Generally, lymph nodes involvement has been shown to predict for increased local and distant recurrence, as well as higher breast cancer mortality [59]. On basis of the Surveillance, Epidemiology, and End Results registry data, Brent's [60] study found a significant association between large lymph node metastasis size and lower breast cancer-specific survival and overall survival even after controlling for other known prognosis factors including number of involved lymph nodes. Moreover, overweight and obesity are not only linked to breast cancer incidence, but women that are obese also have worse outcomes in terms of recurrence and survival. A clinical trial conducted in German [61] showed that obesity constituted an independent, adverse factor in patients with node-positive primary breast cancer. Women who were obese at the time of diagnosis had a shorter disease-free survival and overall survival as compared to women who were non-obese. Thus, BMI, as a modified risk factor, not only plays a crucial role in the occurrence of breast cancer, but also has adverse impact on the outcome and survival of patients. Similarly, we found that BMI had a great influence on the metastasis of various malignant tumors. For example, Zhihong Gong's case-control study [62], following 752 middle-aged prostate cancer patients, concluded that obesity at the time of diagnosis was associated with an increased risk of developing prostate cancer metastasis, regardless of stage or primary treatment. Changhua Wu's retrospective cohort study [63], enrolling 796 primary papillary thyroid cancer patients, indicated that the increment of BMI in patients was associated with the lymph node metastases, and other clinic-pathological features, such as tumor size, extrathyroidal invasion and so on.

It could be considered that the harm of tumor metastasis to patients should not be underestimated, but the reason was still unclear. Several hypothetical mechanisms could explain the association between obesity and lymph node metastasis in breast cancer. One is that the breast size of obese patients is larger, the adipose tissue is thicker, and the palpation of the primary tumor or enlarged axillary lymph nodes is more difficult. Therefore, the accuracy and sensitivity of ultrasonography, molybdenum target and other examinations will be reduced, leading to the delayed or even missed diagnosis of patients, so tumors often in advanced stage or have metastasized at the time of diagnosis [64]. Estrogen, most produced in adipose tissue, have a high level in obese or overweight women, via the aromatization of androstenedione to estrone and then converts to estradiol. This process would in turn facilitate tumor growth. In addition, leptin levels are also higher in obese individuals than those of normal weight, which related to tumor cell proliferation [65]. Some other adipocytokines, such as IL-6 and TNF- $\alpha$ released by activated macrophage, results in inflammation, which could be partly responsible for breast cancer development [66]. Other potential mechanisms for obesityassociated pathologic differences include higher insulin levels and insulin-like growth factors among obese women, which may increase estrogen levels and lead to higher proliferative rates [67]. Notably, in obese breast cancer patients, if the actual body surface area exceeds 2 $\mathrm{m}^{2}$, dose reductions during adjuvant chemotherapy are frequently applied [68]. Up to $40 \%$ of patients may receive limited chemotherapy doses that are not based on actual body weight to avoid possible side effects and toxicity [69]. Meanwhile, aromatase inhibitors, representing an effective endocrine treatment for hormone receptor positive breast cancer patients, were suspected to be less effective in suppression of estrogen levels enough to prevent recurrence in obese women regardless of menopausal status $[70,71]$. Finally, obesity patients often have some unhealthy lifestyle habits, such as excess saturated fat intake and lack of physical activity, resulting in the accumulation of body acid cholesterol, trans fatty acid and other harmful lipid, which are recognized as risk factors for adverse prognosis of breast cancer.

Several limitations existed in our study. Firstly, BMI was calculated by measuring height and weight at the time of diagnosis, which was objective and avoided information bias to some extent. But long-term weight and body composition changes were not take into account, as well as some other potential modifiers (eg. waist circumference and waist-to-hip ratio) for the relationship of BMI and lymph node metastasis in breast cancer. Secondly, some included articles didn't group BMI according to WHO standards, so the accuracy of the results would be affected in the highest versus lowest BMI meta-analysis. Thirdly, we didn't have access to other key individual-level information except area, menopausal status, and study period, such as race, breast cancer sub-types, ER status, progesterone receptor (PR) status, human epidermal growth factor receptor 2 (HER2) status, and obesity associated risk factors (eg. dietary habits and physical inactivity), to examine the roles of these factors in lymph node metastasis. Finally, the retrospective nature of this meta-analysis could not be ignored, so the results should be interpreted with cautions. 


\section{Conclusions}

In conclusion, BMI significantly increases the lymph node metastasis risk of breast cancer. Overweight and obese breast cancer patients might benefit from adhering to a healthy lifestyle aiming at losing or controlling weight, as part of the comprehensive oncologic therapy. Further original studies are warranted to identify the link of BMI and lymph node metastasis in breast cancer.

\section{Abbreviations}

BMI: Body Mass Index; OR: Odds ratio; PMC: PubMed Central; CNKI: The China National Knowledge Infrastructure; VIP: Chinese Scientific Journals; WanFang: Wanfang Data Knowledge Service Platform; NOS: NewcastleOttawa's Scale; RR: Relative risk; TNBC: Triple-negative breast cancer; IL6: Interleukin-6; TNF-a: Tumor necrosis factor-a; ER: Estrogen receptor

\section{Acknowledgements}

Not applicable.

\section{Authors' contributions}

JY, W drafted the manuscript. JY, W and YN, C participated in the design of the study, acquisition of data and performed the statistical analysis. YN, C and FF, Y carried out the literature quality evaluation. ZG, P and L, L conceived of the study, and participated in its design and coordination, and helped to draft the manuscript and revising it critically for important intellectual content and gave final approval of the version to be published. All authors read and approved the final manuscript.

\section{Funding}

This study was supported by the Cultivating grand for youth key teacher in Higher Education Institutions of Henan province (NO: 2017GGJS012); Natural Science Fund of Henan Province (NO: 182300410303); Science and Technology Key Project of Henan province (NO: 172102310373); National Natural Science Foundation of China (NO: 81001280, 81202277). The funding source played no role in the design, collection, analysis, and interpretation of data and in writing the manuscript.

\section{Availability of data and materials}

The datasets generated and/or analyzed during the current study are available in the manuscript.

\section{Ethics approval and consent to participate}

Not applicable.

\section{Consent for publication}

Not applicable.

\section{Competing interests}

The authors declare that they have no competing interests.

Received: 23 January 2020 Accepted: 11 June 2020

Published online: 29 June 2020

\section{References}

1. Bray F, Ferlay J, Soerjomataram I, et al. Global cancer statistics 2018: GLOBOCAN estimates of incidence and mortality worldwide for 36 cancers in 185 countries. CA Cancer J Clin. 2018;68(6):394-424.

2. He J, Chen W. 2017 Chinese cancer registry annual report. Beijing: People's Medical Publishing House; 2017.

3. Sun KX, Zheng RS, Gu XY, et al. Incidence trend and change in the age distribution of female breast cancer in cancer registration areas of China from 2000 to 2014. Zhonghua Yu Fang Yi Xue Za Zhi. 2018;52(6):567-72.

4. Baeyens-Fernandez JA, Molina-Portillo E, Pollan M, et al. Trends in incidence, mortality and survival in women with breast cancer from 1985 to 2012 in Granada, Spain: a population-based study. BMC Cancer. 2018;18(1):781.

5. Virani S, Chindaprasirt J, Wirasorn K, et al. Breast Cancer incidence trends and projections in northeastern Thailand. J Epidemiol. 2018;28(7):323-30.
6. Williams EP, Mesidor M, Winters K, et al. Overweight and obesity: prevalence, consequences, and causes of a growing public health problem. Curr Obes Rep. 2015;4(3):363-70.

7. Finkelstein EA, Khavjou OA, Thompson $\mathrm{H}$, et al. Obesity and severe obesity forecasts through 2030. Am J Prev Med. 2012;42(6):563-70.

8. Choi S, Kim K, Kim SM, et al. Association of Obesity or weight change with coronary heart disease among young adults in South Korea. JAMA Intern Med. 2018;178(8):1060-8.

9. Ryu S, Frith E, Pedisic Z, et al. Secular trends in the association between obesity and hypertension among adults in the United States, 1999-2014. Eur J Intern Med. 2019;62:37-42.

10. Tuei VC, Maiyoh GK, Ha CE. Type 2 diabetes mellitus and obesity in subSaharan Africa. Diabetes Metab Res Rev. 2010;26(6):433-45.

11. Sullivan PW, Ghushchyan VH, Ben-Joseph R. The impact of obesity on diabetes, hyperlipidemia and hypertension in the United States. Qual Life Res. 2008;17(8):1063-71.

12. Shakiba M, Mansournia MA, Kaufman JS. Estimating Effect of Obesity on Stroke Using G-Estimation: The ARIC study. Obesity (Silver Spring). 2019; 27(2):304-8

13. Lai SW. The relationship between obesity in adolescence and pancreatic cancer in adulthood. Cancer. 2019;125(12):2132.

14. Irani J, Lefebvre $O$, Murat $F$, et al. Obesity in relation to prostate cancer risk: comparison with a population having benign prostatic hyperplasia. BJU Int. 2003;91(6):482-4.

15. Montazeri A, Sadighi J, Farzadi F, et al. Weight, height, body mass index and risk of breast cancer in postmenopausal women: a case-control study. BMC Cancer. 2008;8(1):278-80.

16. Wada K, Nagata C, Tamakoshi A, et al. Body mass index and breast cancer risk in Japan: a pooled analysis of eight population-based cohort studies. Ann Oncol. 2014:25(2):519-254.

17. Chen $Y$, Liu L, Zhou Q, et al. Body mass index had different effects on premenopausal and postmenopausal breast cancer risks: a dose-response meta-analysis with 3,318,796 subjects from 31 cohort studies. BMC Public Health. 2017:17(1):936

18. Kawai $M$, Tomotaki A, Miyata $H$, et al. Body mass index and survival after diagnosis of invasive breast cancer: a study based on the Japanese National Clinical Database-Breast Cancer Registry. Cancer Med. 2016;5(6):1328-40.

19. Chan DS, Vieira AR, Aune $D$, et al. Body mass index and survival in women with breast cancer-systematic literature review and meta-analysis of 82 follow-up studies. Ann Oncol. 2014;25(10):1901-14.

20. Haakinson DJ, Leeds SG, Dueck AC, et al. The impact of obesity on breast cancer: a retrospective review. Ann Surg Oncol. 2012;19(9):3012-8.

21. Loi $\mathrm{S}$, Milne RL, Friedlander ML, et al. Obesity and outcomes in premenopausal and postmenopausal breast cancer. Cancer Epidemiol Biomark Prev. 2005;14(7):1686-91.

22. Cui Y, Whiteman MK, Flaws JA, et al. Body mass and stage of breast cancer at diagnosis. Int J Cancer. 2002:98(2):279-83.

23. DerSimonian R, Laird N. Meta-analysis in clinical trials. Control Clin Trials. 1986;7(3):177-88.

24. Aune D, Saugstad OD, Henriksen T, et al. Maternal body mass index and the risk of fetal death, stillbirth, and infant death: a systematic review and metaanalysis. JAMA. 2014;311(15):1536-46.

25. Bagnardi $\mathrm{V}$, Rota $\mathrm{M}$, Botteri $\mathrm{E}$, et al. Light alcohol drinking and cancer: a meta-analysis. Ann Oncol. 2013;24(2):301-8.

26. Bradford B, Cronin R, McKinlay $\mathrm{C}$, et al. Maternally perceived fetal movement patterns: the influence of body mass index. Early Hum Dev. 2019;140: 104922

27. Begg CB, Mazumdar M. Operating characteristics of a rank correlation test for publication bias. Biometrics. 1994;50(4):1088-101.

28. Egger M, Davey SG, Schneider M, et al. Bias in meta-analysis detected by a simple, graphical test. BMJ. 1997;315(7109):629-34.

29. Zhang X, Shi L, Li J, et al. Relationship between body mass index and lymph node metastasis of breast cancer. J Clin Surg. 2014;22(4):299-300.

30. Biglia N, Peano E, Sgandurra P, et al. Body mass index (BMI) and breast cancer: impact on tumor histopathologic features, cancer subtypes and recurrence rate in pre and postmenopausal women. Gynecol Endocrinol. 2013;29(3):263-7.

31. Hanko-Bauer O, Georgescu R, Coros MF, et al. Correlation between obesity and prognostic/predictive parameters with emphasis on the importance of lymph node metastases in patients with invasive breast carcinoma. Pol J Pathol. 2017;68(1):33-9. 
32. Kaviani A, Neishaboury M, Mohammadzadeh N, et al. Effects of obesity on presentation of breast cancer, lymph node metastasis and patient survival: a retrospective review. Asian Pac J Cancer Prev. 2013;14(4):2225-9.

33. Keskin $\mathrm{O}$, Aksoy $\mathrm{S}$, Babacan $\mathrm{T}$, et al. Impact of the obesity on lymph node status in operable breast cancer patients. J BUON. 2013;18(4):824-30.

34. Porter GA, Inglis KM, Wood LA, et al. Effect of obesity on presentation of breast cancer. Ann Surg Oncol. 2006;13(3):327-32.

35. Ewertz $M$, Jensen MB, Gunnarsdottir KA, et al. Effect of obesity on prognosis after early-stage breast cancer. J Clin Oncol. 2011;29(1):25-31.

36. Herlevic VC, Mowad R, Miller JK, et al. Breast cancer outcomes in a population with high prevalence of obesity. J Surg Res. 2015;198(2):371-6.

37. Neuhouser ML, Aragaki AK, Prentice RL, et al. Overweight, obesity, and postmenopausal invasive breast Cancer risk: a secondary analysis of the Women's Health Initiative randomized clinical trials. JAMA Oncol. 2015; 1(5):611-21.

38. Berclaz G, Li S, Price KN, et al. Body mass index as a prognostic feature in operable breast cancer: the international breast Cancer study group experience. Ann Oncol. 2004;15(6):875-84

39. Garrisi VM, Tufaro A, Trerotoli P, et al. Body mass index and serum proteomic profile in breast cancer and healthy women: a prospective study. PLoS One. 2012;7(11):e49631.

40. Mazzarella L, Disalvatore D, Bagnardi V, et al. Obesity increases the incidence of distant metastases in oestrogen receptor-negative human epidermal growth factor receptor 2-positive breast cancer patients. Eur J Cancer. 2013:49(17):3588-97.

41. Smith A, Mullooly M, Murphy L, et al. Associations between obesity, smoking and lymph node status at breast cancer diagnosis in the prostate, lung, colorectal and ovarian (PLCO) Cancer screening trial. PLoS One. 2018; 13(8):e0202291.

42. Wang K, Wu YT, Zhang X, et al. Clinicopathologic and prognostic significance of body mass index (BMI) among breast Cancer patients in Western China: a retrospective multicenter cohort based on Western China clinical cooperation group (WCCCG). Biomed Res Int. 2019;2019:3692093.

43. Copson ER, Cutress RI, Maishman T, et al. Obesity and the outcome of young breast cancer patients in the UK: the POSH study. Ann Oncol. 2015; 26(1):101-12.

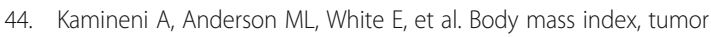
characteristics, and prognosis following diagnosis of early-stage breast cancer in a mammographically screened population. Cancer Causes Control. 2013;24(2):305-12.

45. Mowad R, Chu QD, Li BD, et al. Does obesity have an effect on outcomes in triple-negative breast cancer? J Surg Res. 2013;184(1):253-9.

46. Ademuyiwa FO, Groman A, O'Connor T, et al. Impact of body mass index on clinical outcomes in triple-negative breast cancer. Cancer. 2011;117(18):4132-40

47. Dawood S, Broglio K, Gonzalez-Angulo AM, et al. Prognostic value of body mass index in locally advanced breast cancer. Clin Cancer Res. 2008;14(6):1718-25.

48. Yazici $\mathrm{O}$, Aksoy $\mathrm{S}$, Sendur MA, et al. The effect of obesity on recurrence pattern in early breast cancer patients. J BUON. 2015;20(4):954-62.

49. Schmitz KH, Neuhouser ML, Agurs-Collins T, et al. Impact of obesity on cancer survivorship and the potential relevance of race and ethnicity. J Natl Cancer Inst. 2013;105(18):1344-54.

50. lqbal J, Ginsburg O, Rochon PA, Sun P, Narod SA. Differences in breast cancer stage at diagnosis and cancer-specific survival by race and ethnicity in the United States. JAMA. 2015;313(2):165-73.

51. Qiao X, Xu D, Sun D, Sun S, Huang Z, Cui W. Association analysis of interleukin-18 gene promoter region polymorphisms and susceptibility to sporadic breast cancer in Chinese Han women. J Clin Lab Anal. 2018;32(9):e22591.

52. Simone V, D'Avenia M, Argentiero A, et al. Obesity and breast Cancer: molecular interconnections and potential clinical applications. Oncologist. 2016;21(4):404-17.

53. Rose DP, Vona-Davis L. Biochemical and molecular mechanisms for the association between obesity, chronic inflammation, and breast cancer. Biofactors. 2014;40(1):1-12.

54. Sahin S, Erdem GU, Karatas F, et al. The association between body mass index and immunohistochemical subtypes in breast cancer. Breast. 2017:32:227-36.

55. Pierobon M, Frankenfeld CL. Obesity as a risk factor for triple-negative breast cancers: a systematic review and meta-analysis. Breast Cancer Res Treat. 2013;137(1):307-14.
56. Dent R, Trudeau M, Pritchard Kl, et al. Triple-negative breast cancer: clinical features and patterns of recurrence. Clin Cancer Res. 2007;13:4429-34.

57. Yoo K, Tajima K, Park S, et al. Postmenopausal obesity as a breast cancer risk factor according to estrogen and progesterone receptor status (Japan). Cancer Lett. 2001;167(1):57-63.

58. Gillespie EF, Sorbero ME, Hanauer DA, et al. Obesity and angiolymphatic invasion in primary breast cancer. Ann Surg Oncol. 2010;17(3):752-9.

59. McGale P, Taylor C, Correa C, et al. Effect of radiotherapy after mastectomy and axillary surgery on 10-year recurrence and 20-year breast cancer mortality: meta-analysis of individual patient data for 8135 women in 22 randomised trials. Lancet. 2014;383(9935):2127-35.

60. Rose BS, Jiang W, Punglia RS. Effect of lymph node metastasis size on breast cancer-specific and overall survival in women with node-positive breast cancer. Breast Cancer Res Treat. 2015;152(1):209-16.

61. Scholz C, Andergassen U, Hepp P, et al. Obesity as an independent risk factor for decreased survival in node-positive high-risk breast cancer. Breast Cancer Res Treat. 2015;151(3):569-76.

62. Gong Z, Agalliu I, Lin DW, et al. Obesity is associated with increased risks of prostate cancer metastasis and death after initial cancer diagnosis in middle-aged men. Cancer. 2007;109(6):1192-202.

63. Wu C, Wang L, Chen W, et al. Associations between body mass index and lymph node metastases of patients with papillary thyroid cancer: a retrospective study. Medicine (Baltimore). 2017;96(9):e6202.

64. Arndt V, Stürmer T, Stegmaier C, et al. Patient delay and stage of diagnosis among breast cancer patients in Germany -- a population based study. Br J Cancer. 2002;86(7):1034-40.

65. Geisler J, Haynes B, Ekse D, et al. Total body aromatization in postmenopausal breast cancer patients is strongly correlated to plasma leptin levels. J Steroid Biochem Mol Biol. 2007;104(1-2):27-34.

66. Olefsky JM, Glass CK. Macrophages, inflammation, and insulin resistance. Annu Rev Physiol. 2010;72(1):219-46.

67. Goodwin PJ, Ennis M, Pritchard Kl, et al. Fasting insulin and outcome in early-stage breast cancer: results of a prospective cohort study. J Clin Oncol. 2002;20(1):42-51.

68. Madarnas Y, Sawka CA, Franssen E, Bjarnason GA. Are medical oncologists biased in their treatment of the large woman with breast cancer? Breast Cancer Res Treat. 2001;66(2):123-33.

69. Griggs JJ, Mangu PB, Anderson H, et al. Appropriate chemotherapy dosing for obese adult patients with cancer: American Society of Clinical Oncology clinical practice guideline. J Clin Oncol. 2012;30(13):1553-61.

70. Pfeiler G, Königsberg R, Fesl C, et al. Impact of body mass index on the efficacy of endocrine therapy in premenopausal patients with breast cancer: an analysis of the prospective ABCSG-12 trial. J Clin Oncol. 2011;29(19):2653-9.

71. Folkerd EJ, Dixon JM, Renshaw L, A'Hern RP, Dowsett M. Suppression of plasma estrogen levels by letrozole and anastrozole is related to body mass index in patients with breast cancer. J Clin Oncol. 2012;30(24):2977-80.

\section{Publisher's Note}

Springer Nature remains neutral with regard to jurisdictional claims in published maps and institutional affiliations.

\section{Ready to submit your research? Choose BMC and benefit from:}

- fast, convenient online submission

- thorough peer review by experienced researchers in your field

- rapid publication on acceptance

- support for research data, including large and complex data types

- gold Open Access which fosters wider collaboration and increased citations

- maximum visibility for your research: over $100 \mathrm{M}$ website views per year

At BMC, research is always in progress.

Learn more biomedcentral.com/submissions 